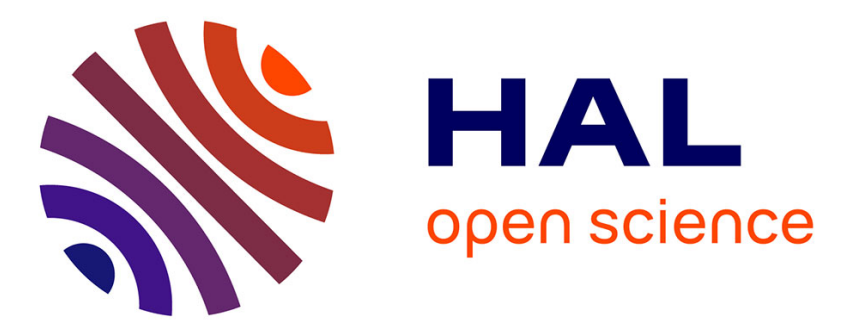

\title{
Anisotropy of non-aqueous layered silicate suspensions subjected to shear flow
}

Christophe Mobuchon, Pierre Carreau, Marie-Claude Heuzey, Naveen Krishna Reddy, Jan Vermant

\section{- To cite this version:}

Christophe Mobuchon, Pierre Carreau, Marie-Claude Heuzey, Naveen Krishna Reddy, Jan Vermant. Anisotropy of non-aqueous layered silicate suspensions subjected to shear flow. Journal of Rheology, 2009, 53 (3), pp.517-538. 10.1122/1.3094911 . hal-01005255

\section{HAL Id: hal-01005255 \\ https://hal.science/hal-01005255}

Submitted on 21 Feb 2017

HAL is a multi-disciplinary open access archive for the deposit and dissemination of scientific research documents, whether they are published or not. The documents may come from teaching and research institutions in France or abroad, or from public or private research centers.
L'archive ouverte pluridisciplinaire $\mathbf{H A L}$, est destinée au dépôt et à la diffusion de documents scientifiques de niveau recherche, publiés ou non, émanant des établissements d'enseignement et de recherche français ou étrangers, des laboratoires publics ou privés. 


\title{
Anisotropy of nonaqueous layered silicate suspensions subjected to shear flow
}

\author{
Christophe Mobuchon, Pierre J. Carreau, and Marie-Claude Heuzey \\ Department of Chemical Engineering, Center for Applied Research on Polymers \\ and Composites (CREPEC), Ecole Polytechnique, P.O. Box 6079, Stn Centre-Ville, \\ Montreal, Quebec, Canada H3C3A7
}

Naveen Krishna Reddy and Jan Vermant

Department of Chemical Engineering, K.U. Leuven, de Croylaan 46, B-3001 Heverlee, Belgium

\begin{abstract}
Nonaqueous layered silicate suspensions have a complex rheological behavior due to the presence of a microstructure on multiple length scales, which is sensitive to flow and flow history. In the present work, the flow-induced orientation and anisotropy of the nonequilibrium metastable structures in nonaqueous layered silicate suspensions has been studied using a combination of light scattering, scattering dichroism, and advanced rheometric measurements, including two dimensional small amplitude oscillatory shear (2D-SAOS) flow experiments. The nature of the structures during flow was mainly studied by means of small angle light scattering patterns. Linear dichroism measurements in the vorticity and velocity gradient directions were used to assess the microstructural anisotropy. The changes observed in the vorticity plane developed in the same range of shear rate as the shear-thinning behavior of the suspensions. Scattering dichroism was used to demonstrate that the flow-induced anisotropy was locked in upon cessation of flow. To verify that this also leads to an anisotropy of the rheological properties, the linear viscoelastic moduli were measured using (2D-SAOS) experiments. This new technique proved to be particularly sensitive to the anisotropic nature of the metastable microstructure of organoclay suspensions. Both the flow-induced orientation and larger scale microstructural rearrangements are shown to contribute to the transient rheological response of the nonaqueous layered silicate suspensions.
\end{abstract}

\section{INTRODUCTION}

Colloidal suspensions exhibit a wide range of complex rheological properties including transition from fluidlike to solidlike behavior leading to nonlinear rheological properties and thixotropic phenomena [Barnes (1997); Mewis (1979)]. This behavior stems from the self-organization of the colloidal particles on multiple length scales [Hoekstra et al. (2005); Pignon et al. (1997a)]. The representation of their inner structure requires description of both spatial and orientation distributions of the dispersed phase over length 
scales ranging from the particle size (a few nanometers) to the macroscopic scale. The understanding of the flow-induced changes in the multiscale structure is relatively well understood for stable colloidal suspensions, whereas for aggregated colloidal suspensions an understanding of flow effects on the size, particle shape, density, and anisotropy of aggregates is only gradually emerging [Vermant and Solomon (2005)].

Layered silicates of the smectite group, commonly used as rheological modifiers in paints, ceramic additives, inks, drilling fluids, and greases, have attracted a great interest in the emerging field of polymer nanocomposites [Patel et al. (2006)]. Their ordering at the nanometer length scale leads to unique mechanical, electrical, optical, and thermal properties [Ray and Okamoto (2003)]. The most commonly used smectite is the montmorillonite for which the individual layers are typically $1.2 \mathrm{~nm}$ thick, 320-400 nm long, and $250 \mathrm{~nm}$ wide [Cadene et al. (2005)]. Nonaqueous suspensions based on layered silicates are expected to belong to the aggregated suspensions class. While many studies have reported the structure of aqueous layered silicate suspensions, there have been fewer investigations of their nonaqueous counterparts. In the following, the extensive literature on aqueous layered silicate suspensions is at first reviewed. The discussion focuses on results pertaining to suspensions of attractive particles.

\section{A. Inorganic smectite suspensions}

Aqueous smectite suspensions undergo a sol-gel transition at medium ionic strength as weakly attractive interactions are induced. In the case of laponite suspensions $(\sim 1 \mathrm{~nm}$ thick, average diameter of $\sim 30 \mathrm{~nm})$, the physical structure of the gel state has been probed using scattering techniques over multiple length scales ranging from the particle size (nanometer) to the micrometer scale [Morvan et al. (1994); Mourchid et al. (1995); Pignon et al. (1998, 1997b); Pignon et al. (1997a); Ramsay and Lindner (1993); Ramsay et al. (1990)]. At small length scales, dense aggregates are formed by the combination of subunits of oriented layers [Morvan et al. (1994); Mourchid et al. (1995); Pignon et al. (1997a)], while at larger scales, of the order of $1 \mu \mathrm{m}$, a fractal network emerges from these dense aggregates [Pignon et al. (1997a)]. The fractal dimension of the network is governed by the physicochemical parameters of the suspension. In turn, the fractal nature of the system controls the rheological behavior of the gel, at least at sufficiently low volume fractions [Pignon et al. (1997a)]. Also the same behavior was observed for model attractive suspensions composed of spheres with fractal-like microstructures [Buscall et al. (1988), Shih et al. (1990)].

Under shear flow conditions, the situation is less clear. The formation of elongated rollers of aggregates arranged perpendicularly to the flow direction has been inferred from scattering experiments by Pignon et al. (1997b, 1998). Indeed, the observed butterfly scattering pattern most likely stems from spatial variations in concentration at length scales that are large compared to the scale of the particles in the direction of the shear flow. The formation of rollerlike structures has been reported from a direct microscopic visualization of attractive emulsions [Montesi et al. (2004)] and for organic carbon nanotube suspensions [Lin-Gibson et al. (2004); Ma et al. (2007)] in a confined shear flow. Beyond a critical shear rate, this characteristic light scattering pattern was replaced by an isotropic one indicating the breakdown of the large anisotropic structure into smaller aggregates [Pignon et al. (1998, 1997b)]. Analogous light scattering patterns were also displayed by organic gels based on spherical particles of silica [Degroot et al. (1994); Hoekstra et al. (2005); Varadan and Solomon (2001); Verduin et al. (1996)], immiscible polymer blends with viscoelastic asymmetry in the melt components [Hobbie et al. (2002)], and semidilute polymer solutions that undergo shear-induced phase separation 
[Moses et al. (1994)]. The mechanism leading to butterfly patterns is attributed by Hoekstra et al. $(2003,2005)$ to the directional dependence of break-up and aggregation of agglomerates, while Hobbie et al. (2004) maintain that it can arise as a consequence of elastic effects. It should be noted that for the aggregated suspensions, only a partial relaxation of the large scale anisotropy was observed after cessation of shear flow [Hoekstra et al. (2005); Mohraz and Solomon (2005); Pignon et al. (1998); Varadan and Solomon (2001); Verduin et al. (1996)]. This denotes the lack of restoring forces to evolve toward isotropy [Mohraz and Solomon (2005); Verduin et al. (1996)].

\section{B. Organic smectite suspensions}

In an organic fluid and depending on its polarity, the inherent attractive interactions between clay layers can be overcome by coating their surface with short hydrocarbons (organomodification). Depending on the strength of the remaining attractive interparticle forces, the resulting organoclay suspensions can remain aggregated and in other cases sterically stabilized Brownian platelets can be obtained. Most often, complete exfoliation is not achieved and a stacking of layers is preserved leading to tactoid structures and aggregates [see for instance Li et al. (2003)]. Small length scale spatial correlations are usually revealed using transmission electron microscopy, scattering techniques including small- and ultrasmall-angle neutron scattering (SANS and USANS) [Hanley et al. (2003); Ho et al. (2001); King et al. (2007); Yoonessi et al. (2005)], and more extensively wideand small-angle X-ray scattering (WAXS and SAXS) [see for instance Vaia et al. (2002)]. To our knowledge, the spatial organization of clay tactoids and aggregates in organic suspensions at large scale have not yet been investigated using light scattering as in the case of aqueous clay gels. Recent investigations using confocal laser microscopy to investigate clay suspensions seem promising for probing their real-space structures [Langat et al. (2006); Yoonessi et al. (2004)].

\section{Objective}

Earlier work by some of the authors [Mobuchon et al. (2007)] showed that the effect of flow history on the rheological properties and structural recovery of organomodified montmorillonite suspensions is complex, with a pronounced dependence on the preshearing history. The aim of the present work is to study the microstructural origin of the rheological response. The orientations of the microstructure and the flow-induced anisotropy are investigated using two-dimensional (2D) static light scattering and linear dichroism measurements under flow. In the system under investigation, changes in the linear dichroism can arise from both form and intrinsic dichroism contributions, hence reflecting aggregate organization and orientation as well as orientation of the individual platelets.

In addition, as in Sung et al. (2008) and Vermant et al. (2007), transient rheological measurements were carried out to probe the microstructure using stress growth measurements in both forward and reverse flows. However, these transient stress measurements are destructive and do not allow a quantification of the structural anisotropy. For this reason, a novel 2D small amplitude oscillatory shear (2D-SAOS) flow method was developed. It has been achieved by synchronizing small amplitude oscillatory motions in directions parallel and orthogonal to the steady shear flow direction. In this way, the linear viscoelastic moduli can be obtained in all the directions of the shear plane leading to a quantitative measure of the microstructure anisotropy. Measurements of the linear viscoelastic properties in the orthogonal direction have been carried out on other systems [De Cleyn and Mewis (1987); Mewis and Schoukens (1978); Simmons (1966); Vermant 
et al. (1998); Walker et al. (2000); Zeegers et al. (1995)]. However, to our knowledge, 2D-SAOS has been used only once to assess the structural anisotropy of lyotropic liquid crystalline polymers [Walker et al. (2000)].

\section{MATERIALS AND METHODS}

\section{A. Materials}

The organoclay suspension used in this investigation is based on an organomodified montmorillonite (Cloisite ${ }^{\circledR} 15$ A, Southern Clay Products). The organophilic clay particles were dispersed in a nonpolar Newtonian blend of polybutenes (Indopol, BP), with a viscosity of $28.5 \mathrm{~Pa} \mathrm{~s}$ at $25^{\circ} \mathrm{C}$. The volume fraction of organoclay, $\phi_{v}$, was fixed at 0.04 , except for the optical measurements that were carried out at lower volume fractions of 0.01 and 0.03 to reduce multiple scattering. Details about the preparation of the suspensions and results from the conventional linear and nonlinear rheometries have been reported elsewhere [Mobuchon et al. (2007)].

\section{B. Optical measurements}

\section{Small angle light scattering}

The parallel plate flow cell of a stress-controlled rheometer Physica MCR300 (Anton Paar) has been combined with a setup for time-resolved flow small angle scattering. A 10 $\mathrm{mW}$ He-Ne laser was used as the light source. The light beam was sent through the sample along the velocity gradient direction by means of a set of prisms. Using a combination of two half convex lenses, a beam stop, and one achromatic lens, the 2D scattering patterns were focused directly onto the chip of a 12-bit IEEE 1394-based digital camera (ORCA-285, Hamamatsu) [Cumming et al. (1992)]. Images were analyzed with in-house developed software (SALSSOFTWARE, KU Leuven). The scattering pattern anisotropy, $A$, is quantified based on the eigenvalues of the second-order tensor of the intensity distribution [Johnson and Fuller (1988)]

$$
A=\frac{\sqrt{\left[\sum I(x, y) x x-\sum I(x, y) y y\right]^{2}+4\left[\sum I(x, y) x y\right]^{2}}}{I},
$$

where $I(x, y)$ is the intensity at the pixel coordinates $(x, y)$ and $I$ is the total intensity. Typically, scattering angles in the light scattering experiments lie between $3^{\circ}$ and $20^{\circ}$. In real space, this corresponds to sizes of approximately $0.5-10 \mu \mathrm{m}(\lambda=632.8 \mathrm{~nm})$.

\section{Linear dichroism}

The optical train used to measure the dichroism [see Fuller (1995) for its definition] is based on a field effect modulator as proposed by Frattini and Fuller (1984). The specific optical train [see Pellens et al. (2005)] consists of a Glan-Thomson polarizer (Newport), a photoelastic modulator (Beaglehole Instruments), and a zero order quarter wave plate (Newport). The modulated light is then sent through the sample by a set of prisms. The transmitted intensity is measured using a sensitive photodiode (Beaglehole Instruments). Its harmonic analysis is performed by two phase lock-in amplifiers (SR830, Stanford Research Systems) to determine the dichroism intensity $\Delta n^{\prime \prime}$ and the orientation angle $\chi$ of its major axes relative to the shear flow direction. Two flow cells mounted on the stress-controlled rheometer (Anton Paar, Physica MCR 300) were used to examine the optical anisotropy in two different planes of the flow field. With the parallel disk flow cell, the light was sent parallel to the velocity gradient direction so that the electric vector sampled the velocity gradient plane. For the Couette cell, the light propagated along the 
TABLE I. Dimensions of the double-walled Couette cell.

\begin{tabular}{ll}
\hline \hline & \\
Inner radius cup $(\mathrm{mm})$ & 22 \\
Inner radius cylinder $(\mathrm{mm})$ & 23 \\
Outer radius cylinder $(\mathrm{mm})$ & 24 \\
Outer radius cup $(\mathrm{mm})$ & 25 \\
Immersed cylinder length $(\mathrm{mm})$ & 50 \\
\hline \hline
\end{tabular}

vorticity axis leading to the measurement of optical anisotropies in the vorticity plane. The parallel disk flow cell consisted of two disks in borosilicate glass. The Couette geometry had an inner bob of $26.7 \mathrm{~mm}$ with a gap of $2 \mathrm{~mm}$ and a sample height of 16.5 $\mathrm{mm}$.

\section{Mechanical measurements}

\section{Transient rheology}

As a first way to mechanically probe the anisotropy of the metastable clay structures, various interrupted forward flow and flow reversal experiments were carried out as in Vermant et al. (2007) and Sung et al. (2008). Tests with and without time delay between the preshearing and the stress growth experiments were performed using a stresscontrolled rheometer (Anton Paar, Physica MCR 501) equipped with a cone-and-plate geometry (radius of $25 \mathrm{~mm}$ and cone angle of $0.04 \mathrm{rad}$ ). Data were considered only after a period of $0.04 \mathrm{~s}$, the time required to reach the specified shear rate within an error of less than $2 \%$.

\section{2D-SAOS}

Conventional linear viscoelastic measurements, following a preshear history, were extended to two dimensions based on methods originally developed for superposition rheometry. Using 2D-SAOS measurements the viscoelastic properties can be obtained in all directions, ranging from parallel to orthogonal directions with respect to the steady shear flow direction. The variations of the moduli with respect to the angle between the previous flow history and the oscillatory flow directly probe and quantify the structural anisotropy. The orthogonal superposition measurements were carried out using a straincontrolled rheometer (ARES, TA Instruments) using a specially designed double-walled Couette cell (dimensions reported in Table I), which is open at the bottom to minimize pumping flows. The bidirectional flow field was imposed by coupling the axial, $\gamma_{z}$, and conventional angular, $\gamma_{\theta}$, oscillatory deformations

$$
\begin{gathered}
\gamma_{r}=0, \\
\gamma_{\theta}=\gamma_{\theta}^{0} \sin (\omega t), \\
\gamma_{z}=\gamma_{z}^{0} \sin \left(\omega t+\delta_{\gamma}\right),
\end{gathered}
$$

with $\omega$ the applied angular frequency. The oscillatory motion is characterized by the relative amplitudes $\gamma_{\theta}^{0}$ and $\gamma_{z}^{0}$, and the phase lag, $\delta_{\gamma}$, between the angular and axial deformations. When the two deformations are in phase, i.e., $\delta_{\gamma}=0$, the resulting oscillatory deformation is unidirectional, with an amplitude given by $\left(\sqrt{\gamma_{\theta}^{0^{2}}+\gamma_{z}^{0^{2}}}\right) / 2$, and oriented at an angle $\phi_{z \theta}=\arctan \left(\gamma_{\theta}^{0} / \gamma_{z}^{0}\right)$ from the perpendicular direction. The resulting stress response is calculated from the components in the $\theta$ and $z$ directions in the same 
manner as the deformation, and its harmonic analysis leads to the viscoelastic moduli at a given angle $\phi_{z \theta}$. With $\delta_{\gamma}=\pi / 2$ and $\gamma_{\theta}^{0}=\gamma_{z}^{0}$, the oscillatory deformation becomes two dimensional, scanning circularly the shear plane. The two-dimensional oscillatory shear with phase lag (2D-SAOS $\delta_{\gamma}$ ) is more suitable for transient measurements since the resulting helicoidal deformation enables a scan of the entire shear plane in a single period. The general observation of the resulting 2D stress gives a direct insight into the structural anisotropy. Moreover, the investigation of the phase lags between the strain and stress components, $\delta_{\theta}$ and $\delta_{z}$, allows discriminating the elastic, $\sigma_{\theta}$, and viscous, $\sigma_{v}$, contributions to the resulting total shear stress, $\sigma$. For an anisotropic viscoelastic material, $\sigma$ can be written as follows:

$$
\begin{gathered}
\sigma_{r}=0, \\
\sigma_{\theta}=\sigma_{\theta}^{0} \sin \left(\omega t+\delta_{\theta}\right), \\
\sigma_{z}=\sigma_{z}^{0} \cos \left(\omega t+\delta_{z}\right) .
\end{gathered}
$$

The viscous and elastic responses are obtained from the in-phase and out-of-phase components, respectively, with respect to the imposed strain

$$
\begin{gathered}
\sigma_{v}=\sqrt{\left[\sigma_{\theta}^{0} \cos \left(\delta_{\theta}\right) \sin (\omega t)\right]^{2}+\left[\sigma_{z}^{0} \cos \left(\delta_{z}\right) \cos (\omega t)\right]^{2}}, \\
\sigma_{e}=\sqrt{\left[\sigma_{\theta}^{0} \sin \left(\delta_{\theta}\right) \cos (\omega t)\right]^{2}+\left[\sigma_{z}^{0} \sin \left(\delta_{z}\right) \sin (\omega t)\right]^{2}} .
\end{gathered}
$$

The corresponding storage and loss moduli can then be obtained by dividing, respectively, $\sigma_{e}$ and $\sigma_{v}$ by the applied strain amplitude, $\gamma^{0}$.

Vibrating the inner cylinder axially provides the oscillatory deformation, $\gamma_{z}$. Its displacement is driven by the normal force rebalanced transducer of the ARES rheometer [Vermant et al. (1997)]. In its regular mode, the ARES rheometer uses a control loop to compensate the displacement of the transducer induced by the normal force. This control loop was modified to impose to the normal force transducer to follow the axial position from the signal supplied by a frequency generator composed of a data card (NI PCI-6221 DAQ, National Intruments) and a LabVIEW (National Instruments) routine. Strain amplitudes in the range of $5 \times 10^{-4}$ up to $5 \times 10^{-2}$ could be applied. The pumping flow induced by the axial motion of the inner cylinder was minimized by some openings at the bottom of the wall [Simmons (1966); Vermant et al. (1997)]. These openings allow the fluid squeezed under the inner cylinder to flow back and forth between the gaps of the cylinders.

The angular oscillatory deformation, $\gamma_{\theta}$, was achieved by rotating the outer cylinder with the servo-controlled motor of the ARES rheometer. This motor was also controlled by the same LabVIEW-commanded frequency generator to ensure synchronization of the axial and angular deformations.

The resulting force and torque signals were measured directly on their respective control board and analyzed using a LabVIEW routine based on the fast Fourier transform for the frequency analysis. The contributions from the instrument (inertia, friction, and elasticity of the transducers) and the sample inertia on the force and torque signals were sufficiently small and did not need to be taken into account (results shown below for the neat polybutene). Measurements for the neat polybutene were conducted with axial and angular deformations of 0.025 - clearly in the linear response region. Smaller deformation amplitudes of 0.01 were used for the organoclay suspensions, chosen such that the response was linear. 
All the previous optical and mechanical measurements were carried out at ambient conditions $\left(\approx 20^{\circ} \mathrm{C}\right)$, except for the transient nonlinear measurements that were done at $25^{\circ} \mathrm{C}$. Prior to the 2D-SAOS measurements, the samples were presheared under various shear rates until a steady-state viscosity was observed and then allowed to rest during $5400 \mathrm{~s}$. The time required to reach steady state depended on the applied shear rate and would be much longer at very low shear rates. However, steady state was reached much faster by sweeping from high to low shear rates [see Mobuchon et al. (2007)]. The reproducibility of the data was estimated to be within $\pm 2 \%$ and $\pm 9 \%$ for shear rates of 25 and $0.001 \mathrm{~s}^{-1}$, respectively. For dynamic measurements, the standard deviation was estimated to be within $\pm 5 \%$.

\section{RESULTS}

\section{A. Flow-induced anisotropy of nonequilibrium and metastable organoclay structures}

\section{Small angle light scattering}

Small angle light scattering (SALS) measurement allows probing the microscale structural evolution under shear flow. After carefully loading the material between the plates, the scattering pattern was found to be isotropic (results not shown). As the length scales probed in the SALS experiments are much larger than the particle size, the power-law decay of the scattered intensity, $I$, with the scattering vector, $q$, suggests that the microstructure is self-similar in nature, in agreement with previous measurements on similar systems [Schaefer et al. (1984); Pignon et al. (1997a)]. The power-law exponent can be associated with a fractal dimension, $D_{f}$, that is found to be of order 2 in the quiescent state, after sample loading. Subjecting the clay suspensions to shear flow resulted in anisotropic SALS patterns. For shear rates ranging from 0.1 to $100 \mathrm{~s}^{-1}$ the isocontour plots are shown in Fig. $1\left(\phi_{v}=0.03\right.$, ambient temperature, exposure time $\left.0.1 \mathrm{~s}\right)$. Under shear flow, a slight anisotropy appears, with two apparent lobes in the scattering patterns running along the flow direction. In real space this corresponds to a more pronounced structure formation along the vorticity axis, as compared to the flow direction. No major changes of this anisotropy with increasing shear rate were observed using the anisotropy factor $A$ based on the eigenvalues of the second-order tensor of Eq. (1) (results not shown). It should be mentioned that this method is not very sensitive to minor changes in anisotropy [Hoekstra et al. (2005)] and results using the more sensitive linear scattering dichroism method are presented in Sec. III A 2. Averages of the intensity as a function of the magnitude of the scattering vector of the patterns in arc segments oriented along the flow and vorticity directions are depicted in Fig. 1 (the curves for the different shear rates have been offset vertically for clarity). The power-law exponent is nearly unchanged, with values of 1.71 and 1.70 when the pattern was scanned along the flow and vorticity directions, respectively. A negligible standard deviation of 0.08 was obtained for the shear rate range explored from 0 to $100 \mathrm{~s}^{-1}$. The essentially constant slope of $I$ versus $q$ with shear rate is in contrast with the reports on more significant shear-induced densification for thermoreversible aggregated suspensions [Hoekstra et al. (2003); Rueb and Zukoski (1997); Varadan and Solomon (2001)]. On the other hand, the presence of a network of mass-fractal clusters is consistent with the low-frequency plateau storage modulus reported earlier [Mobuchon et al. (2007)]. The $I$ versus $q$ curves for the suspension scanned along the two perpendicular directions do differ in intensity, especially at the higher shear rates. Averages on the scattering pattern along the flow direction yield higher intensities. This is in agreement with the reports of rollerlike structures oriented along the vorticity direction in a number of systems [Vermant and Solomon (2005)]. The 


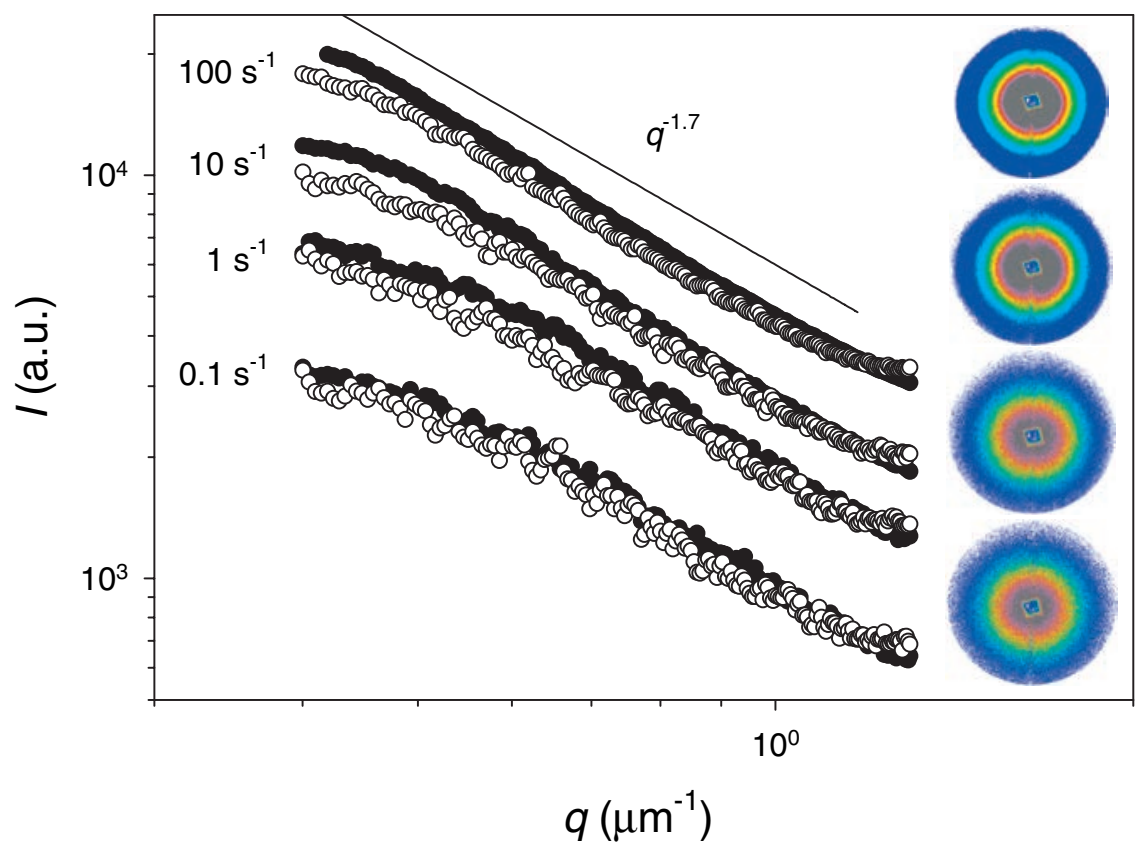

FIG. 1. Scattered intensity distribution $I(q)$ in the flow (filled symbols) and vorticity (unfilled symbols) planes as $\dot{\gamma}$ is varied from 0.1 to $100 \mathrm{~s}^{-1}$. Data are offset for clarity. Solid line represents the measured fractal dimension. Inset figures show the corresponding SALS patterns in the same order as the scattered intensity distributions. The flow direction is from left to right; the vorticity direction is from bottom to top; $\phi_{v}=0.03$.

scattering results suggest that the structure in the flow direction is being broken up by flow. The independence of the fractal dimension of the clusters does suggest that for the present systems the building blocks are not fundamentally altered in the shear range probed. The characteristic cluster size could not be extracted from the intensity distribution since the $I$ vs $q$ does not deviate enough from the power-law behavior in the experimental $q$ range. The picture emerging from the SALS patterns is that of a network of clusters, being broken up by flow. Observations in the real space using confocal laser scanning microscopy will be reported in a subsequent article to further confirm the existence of a mass-fractal network and its dimensions.

\section{Dichroism measurements}

To quantify the flow-induced anisotropy, the dichroism, $\Delta n^{\prime \prime}$, and its orientation angle, $\chi$, were measured for shear rates ranging from $10^{-3}$ to $10^{2} \mathrm{~s}^{-1}$. Figure 2 reports the dichroism, $\Delta n^{\prime \prime}$, with the light propagating along the velocity gradient direction as a function of the applied shear rate for two different volume fractions of particles $\left(\phi_{v}\right.$ $=0.01$ and 0.03 ) and gaps (590 and $949 \mu \mathrm{m}$ with $\left.\phi_{v}=0.03\right)$. The two volume fractions were chosen to be above the liquid-solid transition. At the largest volume fraction of 0.03 the dichroism is shown to be independent of gap size (circular symbols in Fig. 2), indicating that there is no effect of multiple scattering [Batchelor et al. (1987)] and that the maximum length scale of the structures is at least smaller than $590 \mu \mathrm{m}$, as otherwise gap effects should appear. The magnitude of the dichroism increases with the clay volume fraction, but in both cases the same trend with increasing shear rate is displayed, from a low to a high plateau value. 


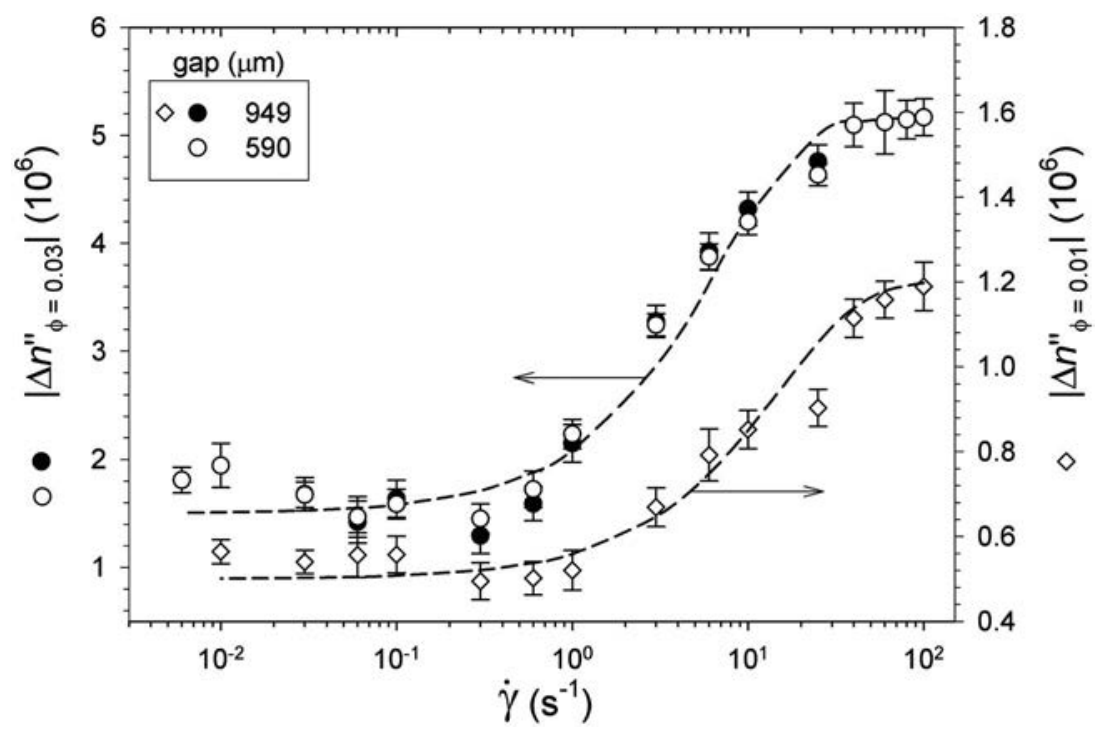

FIG. 2. Effect of the gap and volume fraction on $\left|\Delta n^{\prime \prime}\right|$ measured in the velocity gradient plane as $\dot{\gamma}$ is varied; $\phi_{v}=0.03$

The flow-induced dichroism measured when sending the light along the velocity gradient direction (filled symbols) and the vorticity direction (open symbols) represents the projection of the microstructural anisotropy in the velocity gradient and vorticity planes, respectively. They are given as functions of the applied shear rate in Fig. 3 for a particle volume fraction of 0.01 . The steady-shear viscosity data are also reported on the same figure as the solid lines. In comparison to the results obtained when sending the light along the velocity gradient direction, the dichroism in the vorticity plane exhibits a stronger dependency on the shear rate [Fig. 3(a)]. At the lowest shear rate investigated $\left(10^{-3} \mathrm{~s}^{-1}\right)$, it is nearly zero but increases monotonically with shear rate to a plateau value, just slightly lower than in the velocity gradient plane. As shown in Fig. 3(b), the orientation angle in the vorticity plane (open symbols) also depends on the shear rate, unlike that in the velocity gradient plane, where the orientation angle of the structure is close to $0^{\circ}$ [filled symbols in Fig. 3(b)]. As expected scattering objects, projected onto the velocity gradient plane, are organized along the flow direction. The orientation angle in the vorticity plane (open symbols) is expected to start from $45^{\circ}$ with respect to the flow direction; yet due to measurement accuracy the orientation can be measured from about $25^{\circ}$ at the lowest shear rates and evolves toward the flow direction $\left(0^{\circ}\right)$ at high shear rates. Apart from their different dependencies on shear rate, the changes in the dichroism in the two planes do not take place on the same shear rate range. $\Delta n^{\prime \prime}$ and $\chi$ in the vorticity plane trace the evolution of the shear thinning of the viscosity of the suspension. On the other hand, $\Delta n^{\prime \prime}$ in the velocity gradient plane keeps increasing, although the viscosity plateau at high shear rates is almost reached. The observed evolution of the scattering dichroism cannot be explained solely by the orientation of individual clay particles. Clearly a more complex interplay between the aggregate structure at low shear rates and the orientation of the tactoids or platelets at high shear rates leads to a more complex dependency of the scattering dichroism.

The flow-induced dichroism was observed not to relax upon cessation of flow, and this in both planes (results not shown), at least on the time interval required for the recovery 


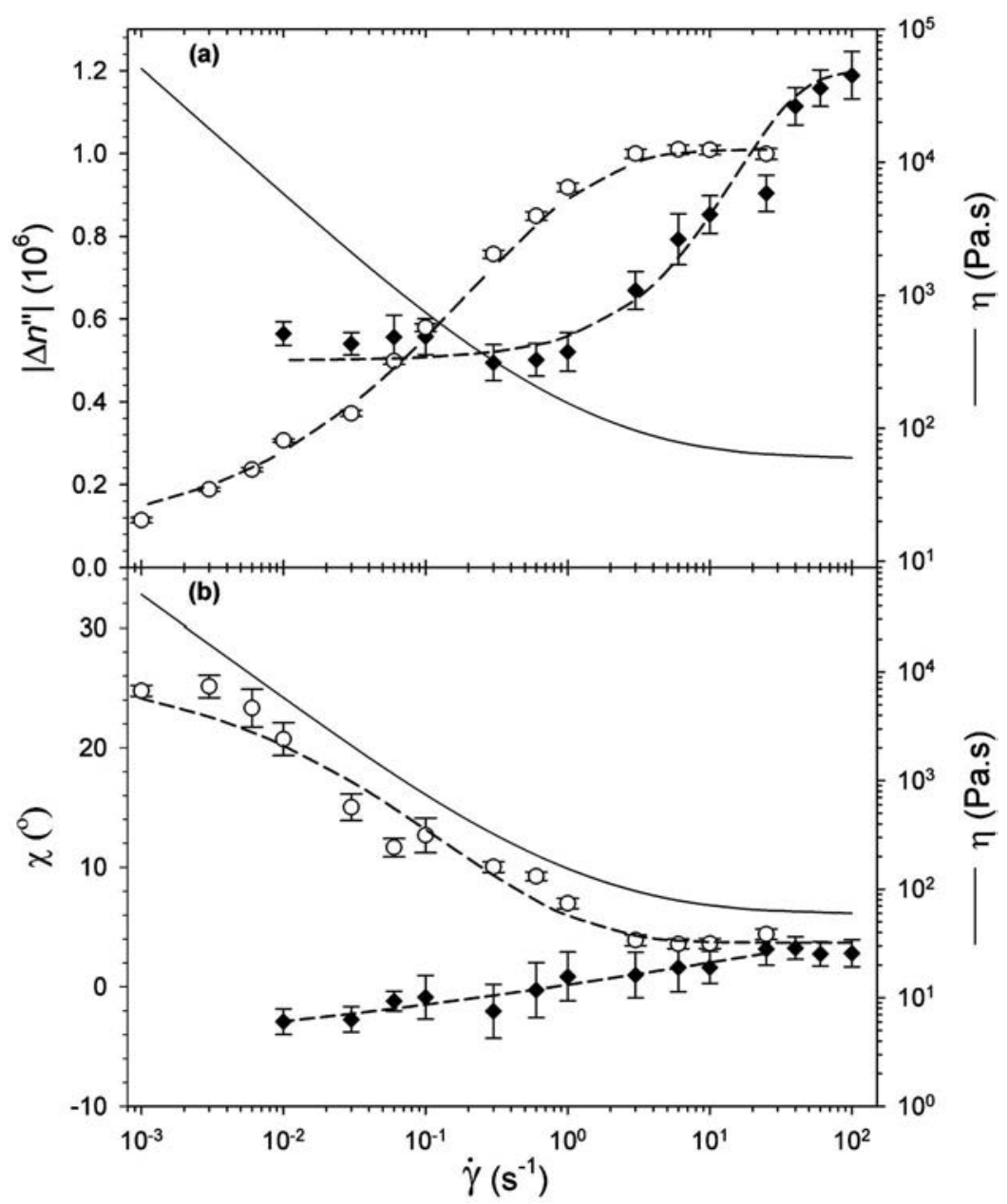

FIG. 3. Dependence of (a) $\left|\Delta n^{\prime \prime}\right|$ and (b) $\chi$ with $\dot{\gamma}$ in the velocity gradient (filled symbols) and vorticity planes (unfilled symbols). $\phi_{v}=0.01$. Dashed lines are regressions to guide the eyes.

of their linear viscoelastic properties [Fig. 2(a) of Mobuchon et al. (2007)]. The flowinduced clay microstructures shown in our previous work are consequently anisotropic and the attractive forces locked in the microstructure. A persisting anisotropy at large scale was also observed for organophilic silica suspensions with the lack of relaxation of their anisotropic SALS patterns [Hoekstra et al. (2005); Mohraz and Solomon (2005); Varadan and Solomon (2001); Verduin et al. (1996)].

\section{B. Orientational contribution to stress growth measurements}

Stress growth experiments are insightful to understand the phenomena of yielding in colloidal gels. Mohraz and Solomon (2005) showed that the stress maximum in start-up experiments can be associated with yielding, using a simple theory of fractal backbone stretching and rupture. In order to evaluate the effect of microstructural anisotropy, stress growth data for forward and reverse directions have been compared. This procedure was initially proposed as a structural-destructive testing to probe the structural anisotropy under flow and at rest [Vermant et al. (2007) and Sung et al. (2008)]. The main features 

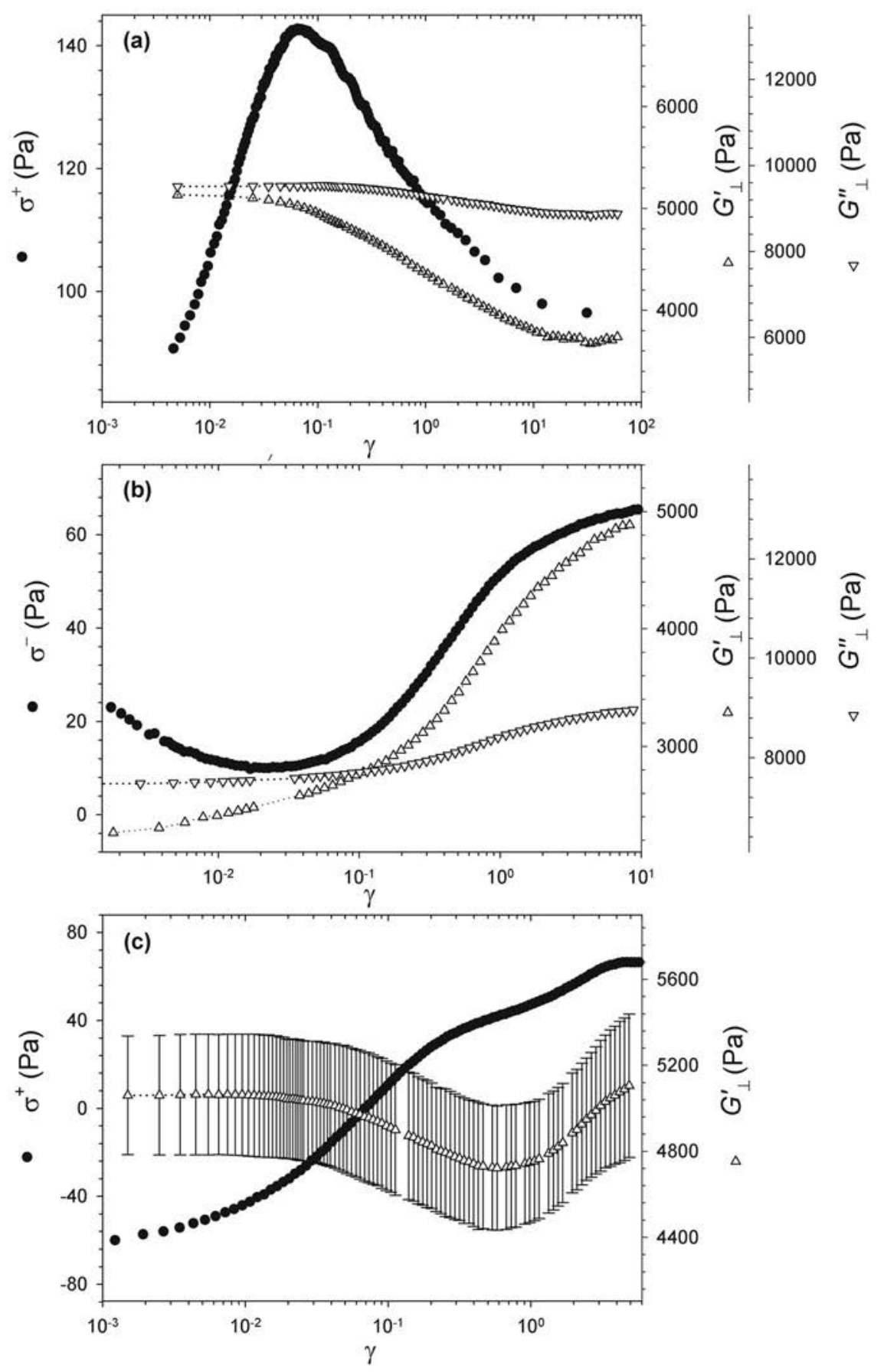

FIG. 4. Typical transient behavior of the organoclay suspension as a function of strain: (a) stepwise increase in shear rate from $\dot{\gamma}_{i}=10^{-3} \mathrm{~s}^{-1}$ to $\dot{\gamma}_{f}=10^{-1} \mathrm{~s}^{-1}$, (b) stepwise decrease in shear rate from $\dot{\gamma}_{i}=1 \mathrm{~s}^{-1}$ to $\dot{\gamma}_{f}$ $=10^{-2} \mathrm{~s}^{-1}$, and (c) reverse flow at $\dot{\gamma}=10^{-2} \mathrm{~s}^{-1} ; \phi_{v}=0.04$. Moduli measured in the orthogonal mode measured at a frequency of $62.8 \mathrm{rad} / \mathrm{s}^{-1}$ during the transient tests are also reported. 
of the transient behavior of the organoclay suspension at a volume fraction of 0.04 are presented in Fig. 4. In addition to the transient shear stress, and to probe the changes in the viscous and elastic contributions to the stress directly, the transient orthogonal moduli $G_{\perp}^{\prime}$ and $G_{\perp}^{\prime \prime}$, measured at a frequency of $62.8 \mathrm{rad} / \mathrm{s}^{-1}$, are reported as a function of the strain, $\gamma$. The moduli were measured using the setup for the axial oscillatory deformation of the 2D-SAOS. Transient superposition measurements are nontrivial to analyze, as the changes in the structure and stress also lead to transients in the oscillatory response. Yet, because of the high frequency used and the relatively long time scales of the transient response, this should not be too problematic in the present case. Hence, we believe this is an adequate tool for probing the evolution of the microstructure. Upon stepwise changes in shear rate, the organoclay suspension undergoes a structure buildup or breakdown. Figure 4(a) illustrates the structure breakdown for a stepwise increase in shear rate from $10^{-3} \mathrm{~s}^{-1}$ to $0.1 \mathrm{~s}^{-1}$. According to Fig. 3(a), this stepwise change in shear rate affects mainly the anisotropy in the vorticity plane. Through the elastic response of the initial structure, the transient shear stress, $\sigma^{+}$, increases as a function of $\gamma$, depicting a typical overshoot before reaching a steady-state value at large strain, as illustrated in Fig. 4(a). The two superposition moduli at this high frequency stay nearly constant during the initial period, which would agree with the simple theory of fractal backbone stretching and rupture of Mohraz and Solomon (2005). Following the maximum in shear stress, which would correspond to the rupture point, the elastic contribution probed by $G_{\perp}^{\prime}$ decreases strongly, combined with a small decrease of $G_{\perp}^{\prime \prime}$ as a function of strain, to reach a less elastic final structure. It should be pointed out that more drastic drops of the superposition moduli are expected at low frequencies. The low frequency part of the spectrum of colloidal suspensions was actually shown experimentally by Potanin et al. (1997) and numerically by Dhont and Wagner (2001) to be more sensitive to the shearinduced structural deformation; yet analyzing the transient response may be tricky. It is clear that the superposition moduli represent direct evidence of the reduction of the elastic contribution to the overall stress, as would be expected following the rupturing of a colloidal network.

A stepwise decrease in shear rate from 1 to $10^{-2} \mathrm{~s}^{-1}$ corresponding to structure buildup is shown in Fig. 4(b). The initial decrease in the shear stress with strain is typical of stress relaxation in viscoelastic materials and the following shear stress increase is due to the structure buildup [Dullaert and Mewis (2005a)]. Both superposition moduli increase, with the elastic component increasing more due to the buildup of the structure. This mimics results from stress jump experiments on weakly aggregated suspensions, where the time evolution of the increase in hydrodynamic and elastic contributions was shown to be very similar [Dullaert and Mewis (2005b)].

To assess the effect of structural anisotropy, the shear stress measured during a reverse flow at $10^{-2} \mathrm{~s}^{-1}$ following an initial forward flow at the same shear rate is reported in Fig. 4(c). At this shear rate, the organoclay network structure, as detected by SALS, is strongly developed and the hydrodynamic contribution to the total steady stress is negligible [see Figs. 3 and 7 of Mobuchon et al. (2007) or the stress jump results from Dullaert and Mewis (2005b)]. In the first part of the curve, where $\sigma^{+}$is negative, the initial structure induced by the preceding shear in the opposite direction remains intact as indicated by the constant $G_{\perp}^{\prime}$. At strains between 0.1 and 1 , a structural reorganization is clearly taking place as can be inferred from the subsequent decrease of the elastic modulus, followed by an increase at larger strains to reach the same value as in the opposite direction.

Tests with and without rest times between the preshearing and the stress growth experiments were also carried out and the results are presented in Fig. 5. Figure 5(a) 


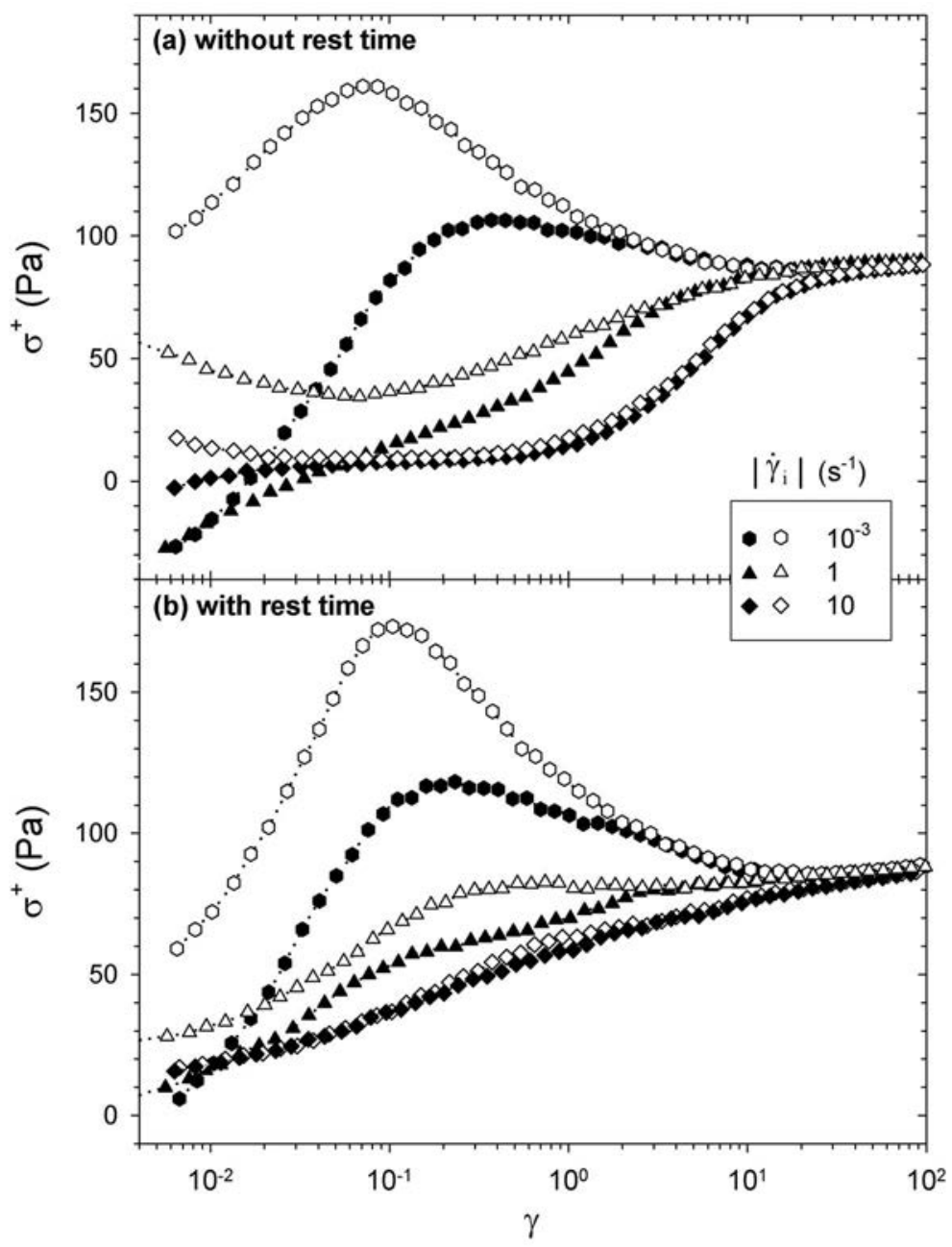

FIG. 5. Stress growth measurements carried out after a preshearing in the same direction (unfilled symbols) and in the opposite direction (filled symbols): (a) after a preshearing at $\left|\dot{\gamma}_{i}\right|$, (b) after preshearing at $\left|\dot{\gamma}_{i}\right|$ and a rest time of $5400 \mathrm{~s} ; \phi_{v}=0.04$.

reports data obtained without a rest time for forward (open symbols) and reverse start-up flow experiments (filled symbols) carried out at an applied shear rate of $\pm 10^{-1} \mathrm{~s}^{-1}$. Figure 5(b) reports the corresponding data obtained following a rest time of $5400 \mathrm{~s}$. In these tests, three different preshear rates, $\dot{\gamma}_{i}$, were used: $10^{-3}, 1$, and $10 \mathrm{~s}^{-1}$. Depending on the amplitude of the preshear rate, the transient shear stresses exhibit either the characteristic evolution described above for a structure breakdown [Fig. 4(a)] or buildup [Fig. 4(b)]. Following a preshearing at $10^{-3} \mathrm{~s}^{-1}$, the forward (open symbols) and reverse (filled symbols) stress growth data of Figs. 5(a) and 5(b) do not overlap, suggesting a flow-induced anisotropy. For a larger preshear rate, the difference between the forward and reverse stress growth data becomes negligible, even after a rest time. These results appear at first to be in contradiction with the increase of anisotropy with shear rate observed previously from the dichroism measurements. This apparent contradiction can 


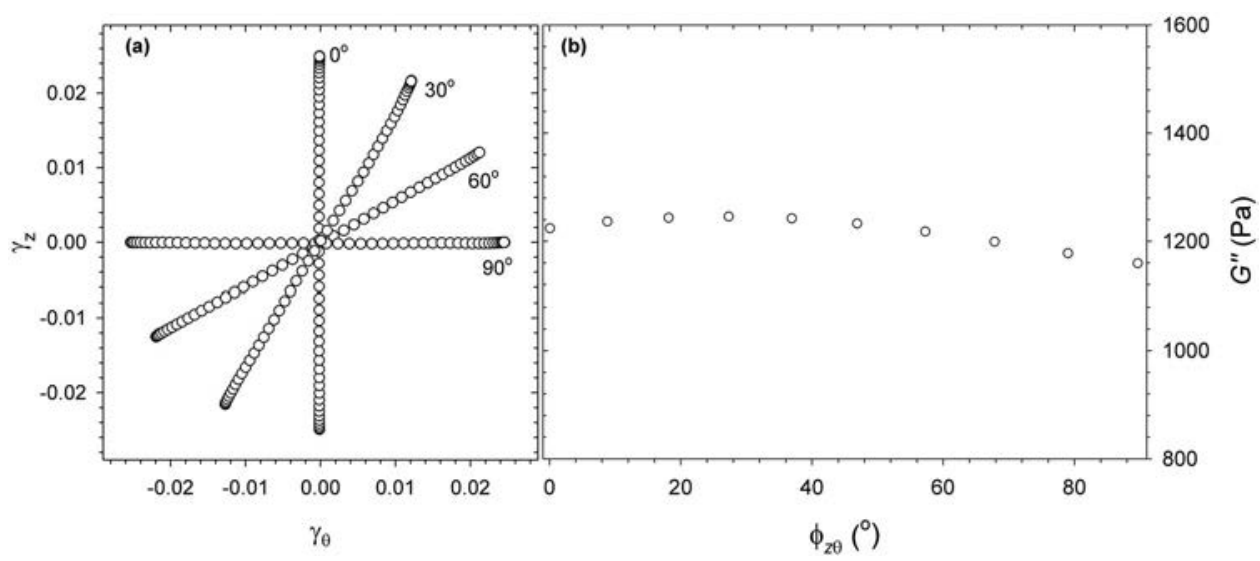

FIG. 6. $2 \mathrm{D}-\mathrm{SAOS}$ at $25 \mathrm{rad} \mathrm{s}^{-1}$ for the Newtonian polybutene suspending fluid: (a) unidirectional oscillatory deformations projected in the shear plane and (b) $G^{\prime \prime}$ as a function of $\phi_{z \theta}$.

be explained by the minor role played by the initial anisotropy on the transient stress response. The evolution of the particle spatial distribution represents instead the main contribution to the resulting structure.

The transient rheological measurements are consistent with the presence of a network that can deform, yield, and subsequently rearrange during flow. The transient rheological measurements combined with the superposition do not yet enable one to come up with a clear view of the structural anisotropy. This is the intent of Sec. III C with 2D-SAOS results.

\section{Mechanical anisotropy of metastable organoclay structures \\ 1. 2D-SAOS}

In these experiments the moduli were probed along a specific direction. At first, the Newtonian suspending fluid (polybutene) was investigated to assess the performance of the setup. Typical unidirectional oscillatory deformations projected in the shear plane are illustrated in Fig. 6(a) for a frequency of $25 \mathrm{rad} \mathrm{s}^{-1}$, in terms of $\gamma_{z}$ vs $\gamma_{\theta}$ for different phase angles, $\phi_{z \theta}$, between the two orthogonal deformations. The oscillatory deformations could be imposed continuously from $0^{\circ}$ to $180^{\circ}$ over a large frequency window. As expected for an isotropic fluid, the loss modulus, $G^{\prime \prime}$, of the Newtonian polybutene does not depend on the shearing angle, $\phi_{z \theta}$ [Fig. 6(b)]. A deviation of less than $\pm 3 \%$ is observed for the $\phi_{z \theta}$ range covered. On the other hand, for the organoclay suspension following a preshear at $3 \mathrm{~s}^{-1}$ and after a rest time of $5400 \mathrm{~s}$, a strong dependency of the moduli with $\phi_{z \theta}$ is observed. Figure 7 shows the experimental results for a frequency of $0.63 \mathrm{rad} / \mathrm{s}^{-1}$. Both moduli vary similarly ( $\delta$ independent of $\phi_{z \theta}$ ) by more than $50 \%$ when the deformations go from perpendicular $\left(\phi_{z \theta}=0^{\circ}\right)$ to the preshearing direction $\left(\phi_{z \theta}\right.$ $=90^{\circ}$ ). Data in the preshearing direction are in good agreement with those previously reported for this system [Fig. 4 of Mobuchon et al. (2007)]. Similar trends are also depicted in Fig. 8 for the elastic modulus [Fig. 8(a)] and for the normalized modulus, $G^{\prime} / G_{\phi_{z \theta}=180^{\circ}}^{\prime}[$ Fig. 8(b)]. We note that the normalized modulus is nearly independent of the frequency. The strong dependency on $\phi_{z \theta}$ outlines the anisotropic nature of the clay structure. It is consistent with the lack of relaxation of the structural anisotropy, as observed by the scattering dichroism after cessation of shear. 


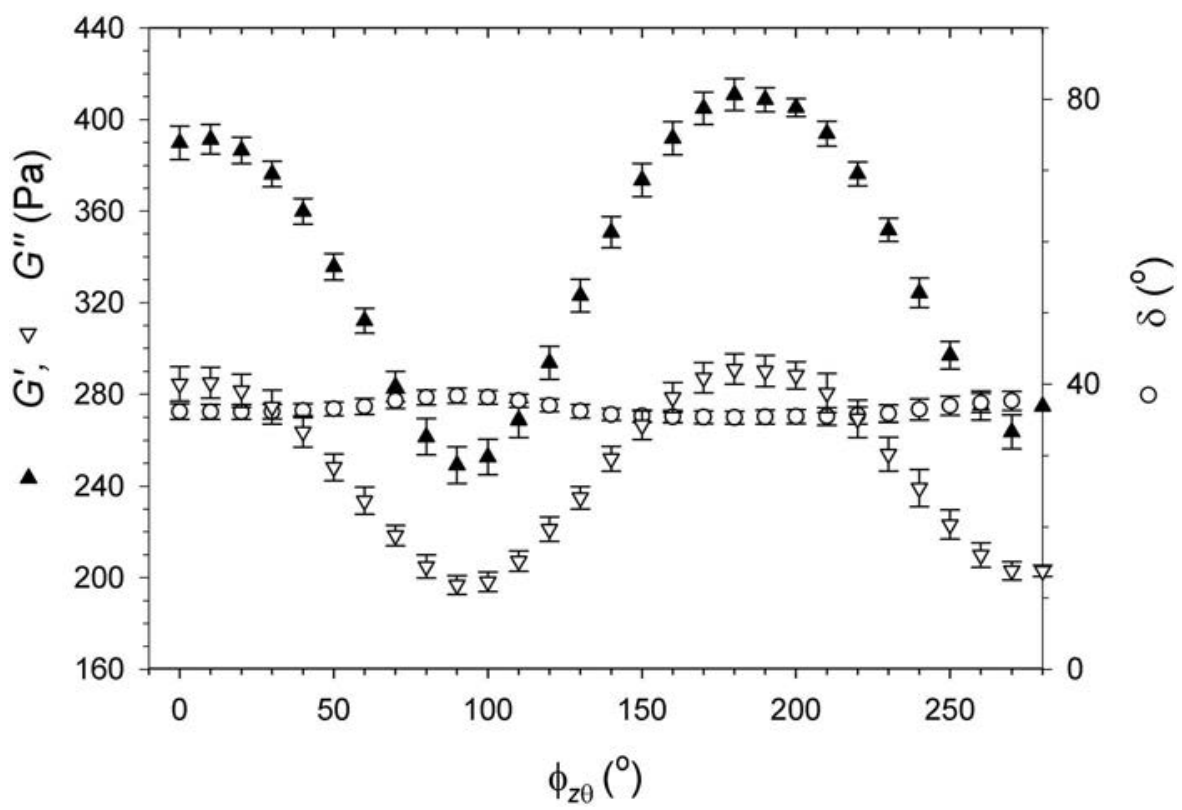

FIG. 7. Viscoelastic 2D-SAOS properties as a function of $\phi_{z \theta}$ at $0.63 \mathrm{rad} \mathrm{s}^{-1}$ after a preconditioning at $3 \mathrm{~s}^{-1}$ and a rest time of $5400 \mathrm{~s}$. The parallel direction of the preshearing corresponds to $\phi_{z \theta}=90^{\circ} ; \phi_{v}=0.04$.

The 2D-SAOS method can be used to investigate the effect of the magnitude of the shear rate on the mechanical anisotropy. Figure 9 presents the effect of the preshearing magnitude, $\dot{\gamma}_{i}$, on the storage modulus of the structure after flow as a function of the solicitation angle at a fixed frequency of $0.6 \mathrm{rad} \mathrm{s}^{-1}$. The storage modulus is observed to decrease with increasing $\dot{\gamma}_{i}$ indicating a weakening of the corresponding final structure [Fig. 9(a)]. However, when normalized as $G^{\prime} / G_{\phi_{z}=180^{\circ}}^{\prime}$, the anisotropy is observed to be independent of the preshearing magnitude $\left(\dot{\gamma}_{i}=3 \times 10^{-3}-3 \mathrm{~s}^{-1}\right)$ and so, for all the different metastable structures reached [Fig. 9(b)]. Note that the structures under flow were found by SALS to be slightly anisotropic in the vorticity and flow directions, but independent of the shear rate (see Fig. 1). Therefore, it seems that the viscoelastic properties, as probed by $2 \mathrm{D}-\mathrm{SAOS}$, are mainly determined by the larger scale structures.

\section{2D-SAOS $\delta_{\gamma}$}

In these experiments the entire shear plane was scanned in a single period, giving an instantaneous "image" of the mechanical anisotropy. The two-dimensional small amplitude oscillatory shear with phase lag was again first tested with the Newtonian polybutene. Figure 10 illustrates the circular applied deformation, $\gamma_{z}$ as a function of $\gamma_{\theta}$, and the resulting stress response, $\sigma_{\theta}$ as a function of $\sigma_{z}$, at a frequency of $25 \mathrm{rad} \mathrm{s}^{-1}$. The stress response is also circular with a slight deformation induced by an added phase shift between the two stress components. This additional phase shift comes from the system response of the normal force rebalanced transducer, which is not fully adapted to perform this experiment at high frequency (mainly due to inertia). The experimental frequency window was therefore limited to values less than $25 \mathrm{rad} \mathrm{s}^{-1}$. As expected for a purely viscous fluid, the phase lag between the strain and stress components is close to $\pi / 2$ and leads with $\gamma^{0}=0.028$ and $\sigma_{\theta}^{0}=\sigma_{z}^{0}=34 \mathrm{~Pa}$ to the same value of the loss modulus, i.e., $G^{\prime \prime} \approx 1200 \mathrm{~Pa}$, as reported in Fig. 6 . 


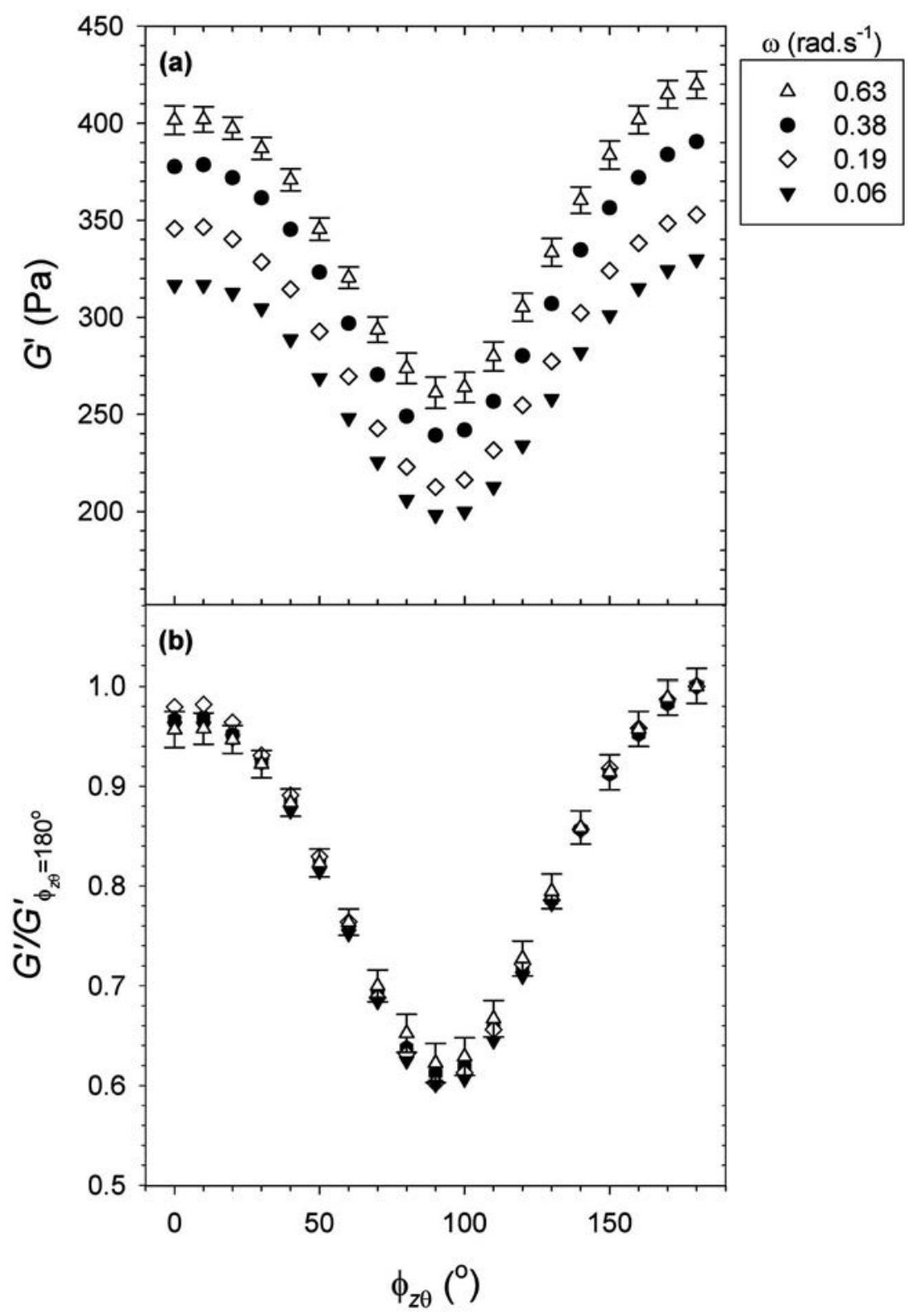

FIG. 8. Frequency dependency of (a) $G^{\prime}$ and (b) $G^{\prime} / G_{\phi_{z \theta-180^{\circ}}}^{\prime}$ as a function of $\phi_{z \theta}$ for the organoclay suspension after a preconditioning at $3 \mathrm{~s}^{-1}$ and a rest time of $5400 \mathrm{~s} ; \phi_{v}=0.04$.

In the case of the organoclay suspension [Fig. 11(a)], the stress response for different preshear rates, $\dot{\gamma}_{i}$, becomes more elliptical as $\dot{\gamma}_{i}$ is decreased with its major axis (highest modulus) perpendicular to the preshearing direction. However, when the normalized stresses, $\sigma_{z} / \sigma_{z_{\max }}$ vs $\sigma_{\theta} / \sigma_{z_{\max }}$, are plotted, the responses become independent of the preshearing magnitude. Similar trends were observed for the stress responses for different frequencies (data not shown for conciseness). When normalized stresses were plotted, as seen before for the 2D-SAOS without phase lag [Fig. 8(b)], the response was observed to 


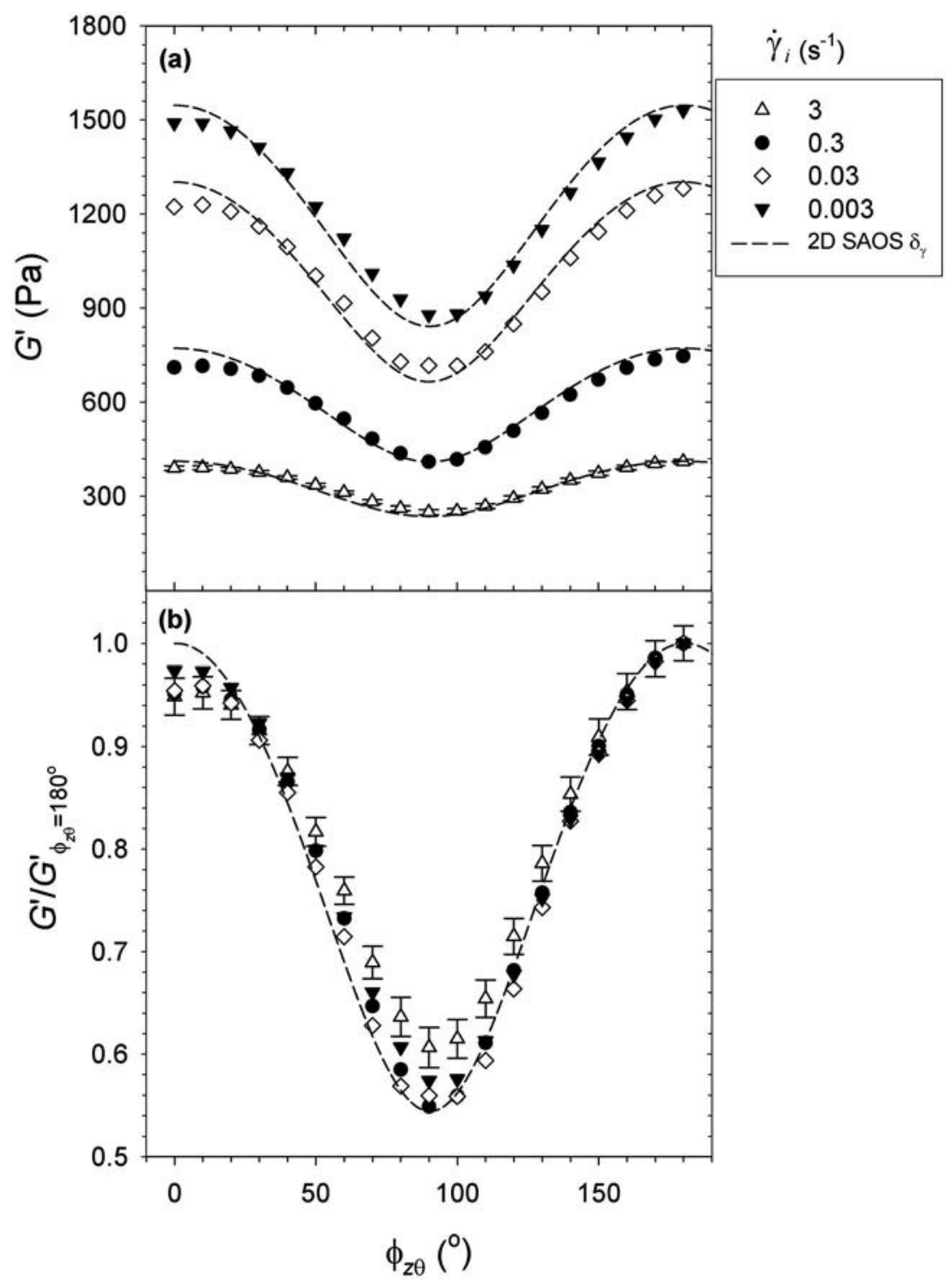

FIG. 9. Flow history dependency of (a) $G^{\prime}$ and (b) $G^{\prime} / G_{\phi_{z \theta=180^{\circ}}}^{\prime}$ for the organoclay suspension as a function of $\phi_{z \theta}$ measured at $0.63 \mathrm{rad} \mathrm{s}^{-1}$ after a rest time of $5400 \mathrm{~s} ; \dot{\phi}_{v}=0.04$.

be independent of the frequency over the range investigated $\left(\omega=0.06-0.63 \mathrm{rad} \mathrm{s}^{-1}\right)$. Finally, the equivalence of these 2D-SAOS $\delta_{\gamma}$ data with 2D-SAOS data obtained without phase lag is shown in Fig. 9(a) by the dashed lines. The storage moduli extracted from the elliptical stresses merge with the ones given by the 2D-SAOS.

\section{CONCLUDING REMARKS}

In this paper the flow-induced anisotropy and orientation of nonaqueous layered silicate suspensions have been probed optically using small angle light scattering and linear dichroism measurements. 2D-SAOS flow has also been proposed as a method to quantify the anisotropy of microstructured materials. For the materials under consideration, a 


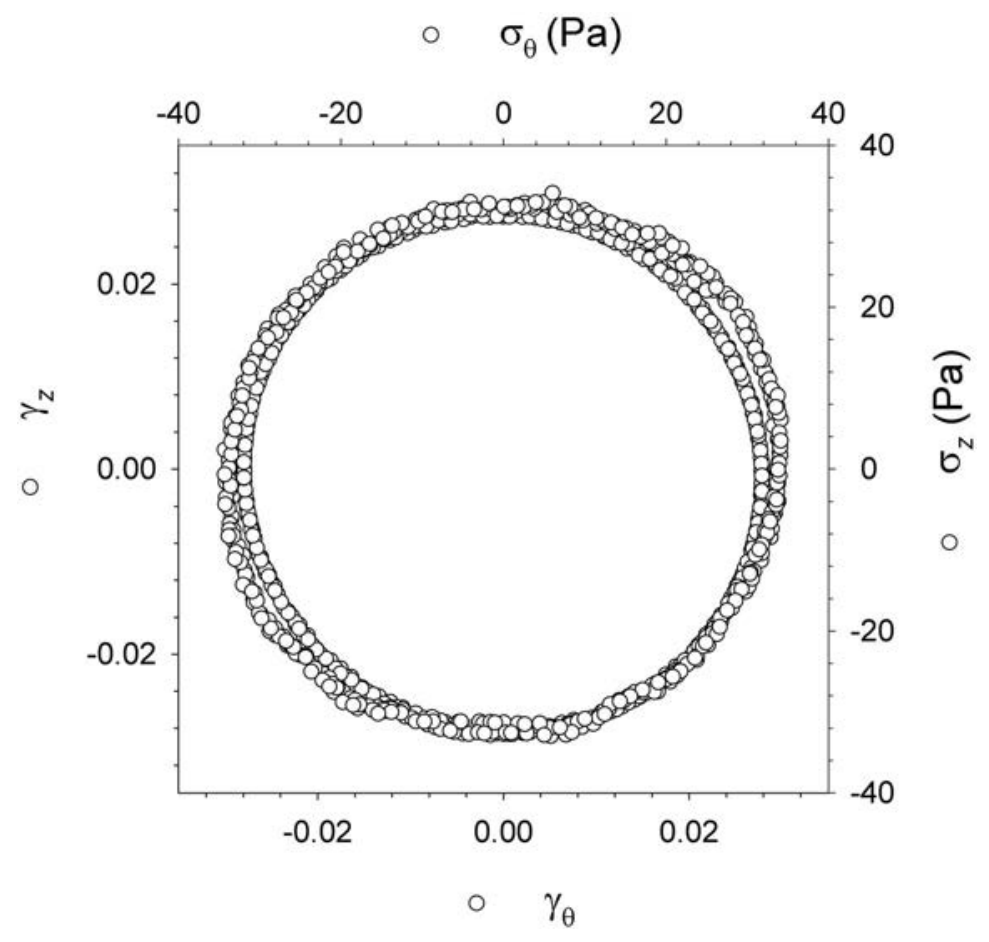

FIG. 10. 2D-SAOS $\delta_{\gamma}$ at $25 \mathrm{rad} \mathrm{s}^{-1}$ for the Newtonian polybutene suspending fluid: $\gamma_{z}$ vs $\gamma_{\theta}$ and $\sigma_{z}$ vs $\sigma_{\theta}$.

network structure, as inferred from the power-law decay of the SALS intensity, is responsible for the observed rheological complexity. During flow, the microstructure becomes anisotropic, with the structure being slightly denser in the flow direction as compared to the vorticity direction. The independence of the power-law slope with shear rate denotes the lack of significant shear-induced densification. Linear scattering dichroism measurements have shown that the anisotropy evolutions in the velocity gradient and vorticity planes do not take place on the same shear rate range. When sending light along the vorticity direction, the monotonic increase of the anisotropy with shear rate toward the flow direction was observed to follow the shear-thinning behavior of the suspension and was ascribed to changes in the aggregate organization, in agreement with the flow SALS observations. In contrast, the anisotropy in the velocity gradient plane still intensifies once the viscosity plateau is reached, accompanied by only a minor evolution of the orientation angle.

Upon cessation of flow, the flow-induced anisotropy in the two directions was observed not to relax due to the presence of attractive interactions, which prevent the systems to evolve toward isotropy. Consequently, the metastable organoclay suspension locks in the flow-induced anisotropy. The 2D-SAOS technique with and without phase lag proved to be sensitive to the anisotropic nature of the organoclay suspensions. As a result of the flow-induced orientation, the transient response of the organoclay suspensions implies both structural and orientational contributions. By analyzing stress growth data, the orientational contribution has been shown to play an increasing role as the transient flow evolves from a structure buildup to a structure breakdown. 2D-SAOS rheometry has been used to show how the elastic and viscous contributions to the stress evolve during transient measurements. 


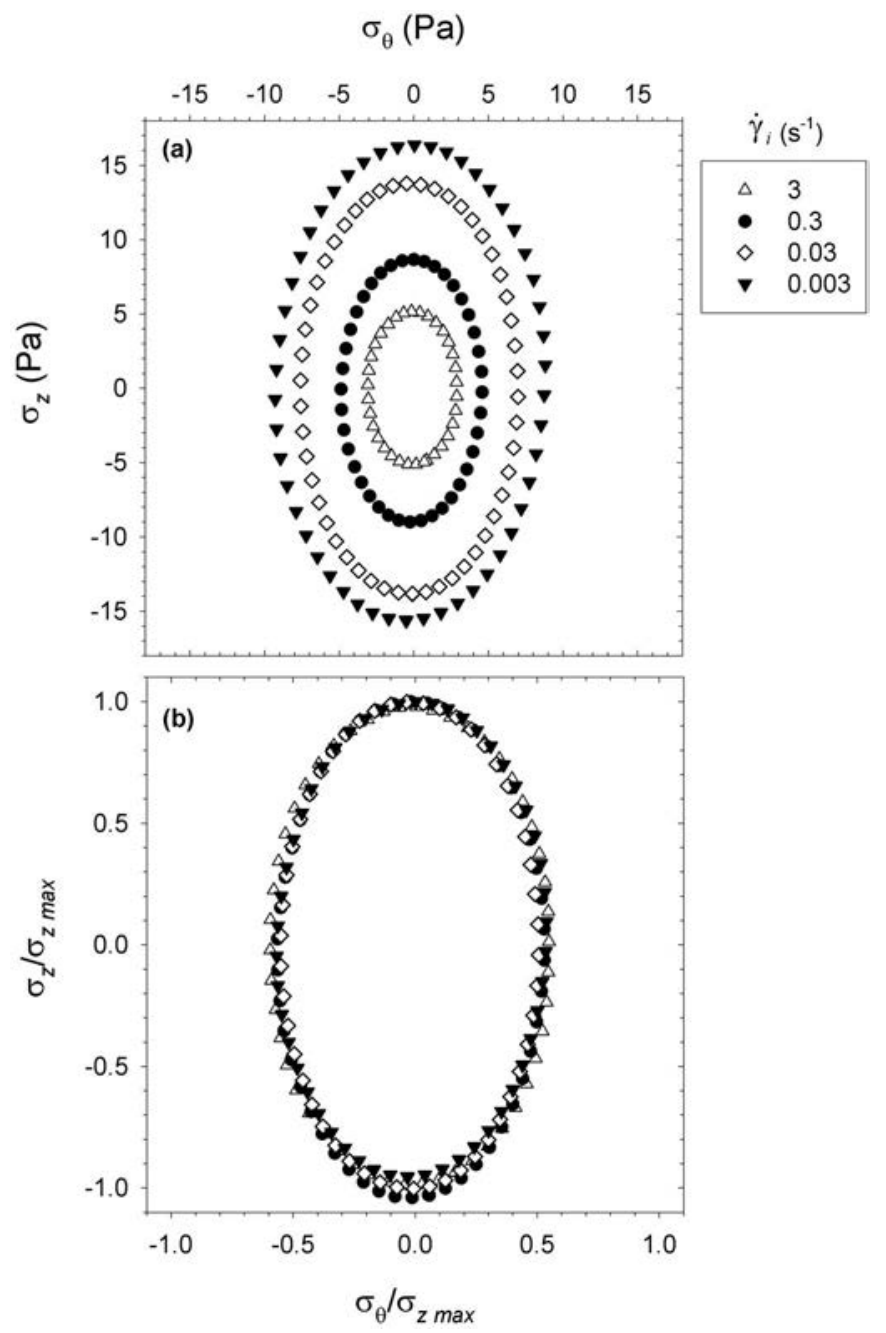

FIG. 11. Flow history dependency of the 2D-SAOS $\delta_{\gamma}$ for the organoclay suspension: (a) $\sigma_{z}$ vs $\sigma_{\theta}$ and (b) $\sigma_{z} / \sigma_{z_{\max }}$ vs $\sigma_{\theta} / \sigma_{z_{\max }}$, measured at $0.63 \mathrm{rad} \mathrm{s}^{-1}$ and after a rest time of $5400 \mathrm{~s} ; \phi_{v}=0.04$.

2D-SAOS technique appears to be powerful, representing an alternative to scattering methods, especially for nontransparent or concentrated complex fluids. The present results are encouraging and should foster further research effort to define the limitations and sensitivity of the 2D-SAOS method.

\section{ACKNOWLEDGMENTS}

The authors wish to thank Allen Glasman (Senior Technical Support Specialist), from TA Instruments, for his precious help in setting up the two-dimensional amplitude oscillatory shear functionality. We also acknowledge helpful support and discussions with Gerald G. Fuller. Southern Clay Products kindly provided the nanoclay used in this study. We are thankful to one of the reviewer for his most helpful comments on the transient behavior of a colloidal network. Finally, financial support from NSERC (Natural Science 
and Engineering Research Council of Canada) and from FQRNT (Fonds Québécois de Recherche en Nature et Technologies) is gratefully acknowledged.

\section{References}

Barnes, H. A., "Thixotropy-A review," J. Non-Newtonian Fluid Mech. 70, 1 (1997).

Batchelor, P., G. H. Meeten, and G. C. Maitland, "Linear birefringence and dichroism measurements on polytetrafluorethylene dispersions," J. Colloid Interface Sci. 117, 360 (1987).

Buscall, R., P. D. A. Mills, J. W. Goodwin, and D. W. Lawson, "Scaling behavior of the rheology of aggregate networks formed from colloidal particles," J. Chem. Soc., Faraday Trans. 84, 4249 (1988).

Cadene, A., S. Durand-Vidal, P. Turq, and J. Brendle, "Study of individual Na-montmorillonite particles size, morphology, and apparent charge,” J. Colloid Interface Sci. 285, 719 (2005).

Cumming, A., P. Wiltzius, F. S. Bates, and J. H. Rosedale, "Light-scattering experiments on phase-separation dynamics in binary fluid mixtures," Phys. Rev. A 45, 885 (1992).

De Cleyn, G. and J. Mewis, "Constitutive equation for polymer liquids: Application to shear flow," J. NonNewtonian Fluid Mech. 9, 91 (1987).

Degroot, J. V., C. W. Macosko, T. Kume, and T. Hashimoto, "Flow-induced anisotropic SALS in silica-filled PDMS liquids,” J. Colloid Interface Sci. 166, 404 (1994).

Dhont, J. K. G. and N. J. Wagner, “Superposition rheology," Phys. Rev. E 63, 021406 (2001).

Dullaert, K. and J. Mewis, "Thixotropy: Build-up and breakdown curves during flow," J. Rheol. 49, 1213 (2005a).

Dullaert, K. and J. Mewis, "Stress jumps on weakly flocculated dispersions: Steady state and transient results," J. Colloid Interface Sci. 287, 542 (2005b).

Frattini, P. L. and G. G. Fuller, "Dynamic of dilute colloidal suspensions subject to time-dependent flow fields by conservative dichroism,” J. Colloid Interface Sci. 100, 506 (1984).

Fuller, G. G., Optical Rheometry of Complex Fluids (Oxford University Press, New York, 1995).

Hanley, H. J. M., C. D. Muzny, D. L. Ho, and C. J. Glinka, "A small-angle neutron scattering study of a commercial organoclay dispersion,” Langmuir 19, 5575 (2003).

Ho, D. L., R. M. Briber, and C. J. Glinka, "Characterization of organically modified clays using scattering and microscopy techniques," Chem. Mater. 13, 1923 (2001).

Hobbie, E. K., H. S. Jeon, H. Wang, H. Kim, D. J. Stout, and C. C. Han, "Shear-induced structure in polymer blends with viscoelastic asymmetry," J. Chem. Phys. 117, 6350 (2002).

Hobbie, E. K., S. Lin-Gibson, H. Wang, J. A. Pathak, and H. Kim, "Ubiquity of domain patterns in sheared viscoelastic fluids," Phys. Rev. E 69, 061503 (2004).

Hoekstra, H., J. Mewis, T. Narayanan, and J. Vermant, "Multi length scale analysis of the microstructure in sticky sphere dispersions during shear flow," Langmuir 21, 11017 (2005).

Hoekstra, H., J. Vermant, and J. Mewis, "Flow-induced anisotropy and reversible aggregation in twodimensional suspensions," Langmuir 19, 9134 (2003).

Johnson, S. J. and G. G. Fuller, "Optical anisotropy of sheared hematite suspensions,” J. Colloid Interface Sci. 124, 441 (1988).

King, H. E., S. T. Milner, M. Y. Lin, J. P. Singh, and T. G. Mason, "Structure and rheology of organoclay suspensions," Phys. Rev. E 75, 021403 (2007).

Langat, J., S. Bellayer, P. Hudrlik, A. Hudrlik, P. H. Maupin, S. J. W. Gilman, and D. Raghavan, "Synthesis of imidazolium salts and their application in epoxy montmorillonite nanocomposites," Polymer 47, 6698 (2006).

Li, J., C. Zhou, G. Wang, and D. Zhao, "Study on rheological behavior of polypropylene/clay nanocomposites," J. Appl. Polym. Sci. 89, 3609 (2003).

Lin-Gibson, S., J. A. Pathak, E. A. Grulke, H. Wang, and E. K. Hobbie, "Elastic flow instability in nanotube suspensions," Phys. Rev. Lett. 92, 048302 (2004).

Ma, A., M. Mackley, and S. Rahatekar, "Experimental observation on the flow-induced assembly of carbon 
nanotube suspensions to form helical bands," Rheol. Acta 46, 979 (2007).

Mewis, J., “Thixotropy-a general review,” J. Non-Newtonian Fluid Mech. 6, 1 (1979).

Mewis, J. and G. Schoukens, "Mechanical spectroscopy of colloidal dispersions," Faraday Discuss. Chem. Soc. 65, 58 (1978).

Mobuchon, C., P. J. Carreau, and M. C. Heuzey, "Effect of flow history on the structure of a non-polar polymer/clay nanocomposite model system," Rheol. Acta 46, 1045 (2007).

Mohraz, A. and M. J. Solomon, "Orientation and rupture of fractal colloidal gels during start-up of steady shear flow," J. Rheol. 49, 657 (2005).

Montesi, A., A. A. Pena, and M. Pasquali, "Vorticity alignment and negative normal stresses in sheared attractive emulsions," Phys. Rev. Lett. 92, 058303 (2004).

Morvan, M., D. Espinat, J. Lambard, and T. Zemb, "Ultrasmall-angle and small-angle x-ray-scattering of smectite clay suspensions," Colloids Surf., A 82, 193 (1994).

Moses, E., T. Kume, and T. Hashimoto, "Shear microscopy of the butterfly pattern in polymer mixtures," Phys. Rev. Lett. 72, 2037 (1994).

Mourchid, A., A. Delville, J. Lambard, E. Lecolier, and P. Levitz, "Phase-diagram of colloidal dispersions of anisotropic charged-particles-Equilibrium properties, structure, and rheology of laponite suspensions," Langmuir 11, 1942 (1995).

Patel, H. A., R. S. Somani, H. C. Bajaj, and R. V. Jasra, "Nanoclays for polymer nanocomposites, paints, inks, greases and cosmetics formulations, drug delivery vehicle and waste water treatment," Bull. Mater. Sci. 29, 133 (2006).

Pellens, L., J. Vermant, and J. Mewis, "Deviations from the stress-optical rule in telechelic associative polymer solutions," Macromolecules 38, 1911 (2005).

Pignon, F., A. Magnin, and J. M. Piau, "Butterfly light scattering pattern and rheology of a sheared thixotropic clay gel," Phys. Rev. Lett. 79, 4689 (1997b).

Pignon, F., A. Magnin, and J.-M. Piau, "Thixotropic behavior of clay dispersions: Combinations of scattering and rheometric techniques,” J. Rheol. 42, 1349 (1998).

Pignon, F., A. Magnin, J. M. Piau, B. Cabane, P. Lindner, and O. Diat, "Yield stress thixotropic clay suspension: Investigations of structure by light, neutron, and x-ray scattering," Phys. Rev. E 56, 3281 (1997a).

Potanin, A. A., S. M. Shrauti, D. W. Arnold, A. M. Lane, and J. Mellema, "Testing the structure of magnetic paints with and without superimposed shear," J. Rheol. 41, 1337 (1997).

Ramsay, J. D. F. and P. Lindner, "Small-angle neutron-scattering investigations of the structure of thixotropic dispersions of smectite clay colloids,” J. Chem. Soc., Faraday Trans. 89, 4207 (1993).

Ramsay, J. D. F., S. W. Swanton, and J. Bunce, "Swelling and dispersion of smectite clay colloidsDetermination of structure by neutron-diffraction and small-angle neutron-scattering," J. Chem. Soc., Faraday Trans. 86, 3919 (1990).

Ray, S. S. and M. Okamoto, "Polymer/layered silicate nanocomposites: A review from preparation to processing," Prog. Polym. Sci. 28, 1539 (2003).

Rueb, C. J. and C. F. Zukoski, "Viscoelastic properties of colloidal gels,” J. Rheol. 41, 197 (1997).

Schaefer, D. W., J. E. Martin, P. Wiltzius, and S. D. Cannell, "Fractal geometry of colloidal aggregates," Phys. Rev. Lett. 52, 2371 (1984).

Shih, W. H., W. Y. Shih, S. I. Kim, J. Liu, and I. A. Aksay, "Scaling behavior of the elastic properties of colloidal gels," Phys. Rev. A 42, 4772 (1990).

Simmons, J. M., "A servo-controlled rheometer for measurement of the dynamic modulus of viscoelastic liquids,” J. Sci. Instrum. 43, 887 (1966).

Sung, J. H., J. Mewis, and P. Moldenaers, "Transient rheological probing of PIB/hectorite-nanocomposites," Korea-Aust. Rheol. J. 20, 27 (2008).

Vaia, R. A. and W. Liu, "X-ray powder diffraction of polymer/layered silicate nanocomposites: Model and practice,” J. Polym. Sci. Part Polym. Phys. 40, 1590 (2002).

Varadan, P. and M. J. Solomon, "Shear-induced microstructural evolution of thermoreversible colloidal gel," Langmuir 17, 2918 (2001).

Verduin, H., B. J. deGans, and J. K. G. Dhont, "Shear induced structural changes in a gel-forming suspension studied by light scattering and rheology," Langmuir 12, 2947 (1996). 
Vermant, J., S. Ceccia, M. K. Dolgovskij, P. L. Maffettone, and C. W. Macosko, "Quantifying dispersion of layered nanocomposites via melt rheology,” J. Rheol. 51, 429 (2007).

Vermant, J., P. Moldenaers, J. Mewis, M. Ellis, and R. Garritano, "Orthogonal superposition measurements using a rheometer equipped with a force rebalanced transducer,” Rev. Sci. Instrum. 68, 4090 (1997).

Vermant, J. and M. J. Solomon, "Flow-induced structure in colloidal suspensions," J. Phys.: Condens. Matter 17, R187 (2005).

Vermant, J., L. Walker, P. Moldenaers, and J. Mewis, "Orthogonal versus parallel superposition measurements," J. Non-Newtonian Fluid Mech. 79, 173 (1998).

Walker, L. M., J. Vermant, P. Moldenaers, and J. Mewis, "Orthogonal and parallel superposition measurements on lyotropic liquid crystalline polymers," Rheol. Acta 39, 26 (2000).

Yoonessi, M., H. Toghiani, T. L. Daulton, J.-S. Lin, and C. U. Pittman, Jr., "Clay delamination in clay/ poly(dicyclopentadiene) nanocomposites quantified by small angle neutron scattering and high-resolution transmission electron microscopy," Macromolecules 38, 818 (2005).

Yoonessi, M., H. Toghiani, W. L. Kingery, and C. U. Pittman, "Preparation, characterization, and properties of exfoliated/delaminated organically modified clay/dicyclopentadiene resin nanocomposites," Macromolecules 37, 2511 (2004).

Zeegers, J., D. Ende, C. Blom, E. G. Altena, G. J. Beukema, and J. Mellema, “A sensitive dynamic viscometer for measuring the complex shear modulus in a steady shear flow using the method of orthogonal superposition," Rheol. Acta 34, 606 (1995). 